\title{
Providing perioperative care for patients with hip fractures
}

\author{
G. T. C. Wong • N. C. H. Sun
}

Received: 31 August 2010 / Accepted: 8 September 2010

(C) The Author(s) 2010. This article is published with open access at Springerlink.com

\begin{abstract}
Providing perioperative care for patients with hip fractures can present major challenges for the anaesthesiologist. These patients often have multiple comorbidities, the deterioration of any one of which may have precipitated the fall. A careful balance has to be achieved between minimising the time before operation and spending time to optimise their medical status. This review will present insights into preoperative patient assessment and optimization in this group of patients from the anaesthesiologists' perspective. In particular, it will highlight important medical issues of concern that may alter anaesthetic risks and management. With a greater understanding of what these issues are, potentially a more prompt and integrated approach to managing these patients may be made. Hopefully, this would result in minimising last minute cancellations due to medical reasons for these patients.
\end{abstract}

Keywords Hip fracture - Osteoporosis · Preoperative anaesthetic assessment

\section{Introduction}

Providing anaesthesia for patients undergoing surgery for their hip fractures is particularly challenging for anaesthesiologists as the patients are usually elderly with multiple comorbidities, the instability in any one of which may have triggered the sentinel event. The urgency of hip fracture surgery usually is not deemed as emergency and yet prolonged delay in the quest for further optimization can paradoxically cause a downward

G. T. C. Wong $(\bowtie) \cdot$ N. C. H. Sun

Department of Anaesthesiology,

University of Hong Kong,

Room K424, Queen Mary Hospital,

Pokfulam, Hong Kong

e-mail: gordon@hku.hk spiral in the patient's general status, as new problems may develop consequent to the continued immobility and pain. Even in patients with significant medical conditions and high anaesthetic risk, request to proceed to surgery can still be justified as surgical treatment is the best form of analgesia and will improve comfort for the patient and facilitate nursing care. Although the reason for surgical delay is usually due to hospital organization or the health care system in the vast percentage of cases, it is particularly frustrating for all involved when the patient's surgery is cancelled at the last minute for medical reasons, especially ones that seem avoidable or even unreasonable. The anaesthesiologist is required to exercise careful judgement in balancing between the risks to the patient against the benefits of early fixation, especially when multiple considerations can impact upon the decision-making pathway. In addition to the certain "knowns" regarding the patient's condition such as physical signs and selected laboratory data, there are also many "unknowns" such as any new or pre-existing neurological symptoms in the uncommunicative or the pre-injury functional capacity in the apparent immobile. Furthermore, there are non-medical considerations such as family or patient expectations, theatre availability, expertise of the operator and anaesthesiologists. This article will discuss risk assessment in hip fracture surgery from the anaesthesiologist's perspective. It will aim to look at common causes for concern from a pathophysiological basis and suggest ways in which we may be able to minimise avoidable last minute cancellation.

\section{Avoiding last minute cancellation begins with the immediate care of patients}

Developing practice guidelines that is adapted to the local institution's conditions may avoid recurrent or preventable 
systematic or medical errors and is the crucial first step in avoiding last minute cancellations. All good care begins obtaining a careful and focused medical history and performing a physical examination. Obtaining and documenting a collaborative history from a carer or witness where possible is invaluable in gaining insight into the precipitating factors for the injury and in determining the timing of the event. Knowing the time of injury and the duration of any immediately preceding illness would enable better interpretation of clinical signs and laboratory results. Patients that were unwell before the injury may already have been developing conditions such as electrolyte imbalances or infections that could delay surgery. Their fluid and nutritional intake could already be impaired and their normal medications may have been omitted. Reduced fluid intake and extravasation into the site of injury can account for substantial fluid deficit, especially in the elderly. Pharmacokinetic as well as pharmacodynamic properties of medications may have been altered due to these changes in the patient's physiological status. Early intervention may arrest further deterioration or even improve the situation. For example, fluid and electrolyte resuscitation should begin immediately after assessment, taking into account the deficits that have already been accumulated since the time of injury and the ongoing requirements from preoperative fasting. Fluid replacement should therefore be more aggressive than providing simple maintenance requirements. It should be guided by electrolyte levels when they come to hand and may benefit from invasive monitoring protocol guidance $[1,2]$.

History suggesting an acute cardiac and cerebral event precipitating the injury should be investigated as soon as possible after admission. It is important to appreciate that factors conducive to the development of myocardial ischemia are present from the time of injury and are not necessarily confined to the operative period. These include suboptimal respiratory ventilation and oxygenation from being immobile in the supine position, increased oxygen demand secondary to pain-induced tachycardia, tachycardia-associated increase in shear stress to coronary atherosclerotic plaques and traumaassociated hypercoagulability [3]. Last but not least, a review and rational plan for the patient's usual medications is paramount to minimise further physiological disturbance to the patient.

\section{Preoperative anaesthetic assessment: what is important?}

The overall purpose for preoperative assessment is to identify those patients which, on the basis of their current physiological status, are more likely to develop postoperative medical complications. Also, it aims to exclude any conditions in patients that are likely to develop into life- threatening events should they experience any further acute physiological stresses. For example, blood loss and fluid shifts needing immediate replacement can quickly induce hemodynamic instability, electrolyte disturbance, oxygen supply and demand imbalances that can lead to acute organ dysfunction such as unstable arrhythmias. This process is commonly misinterpreted by non-anaesthesiologists as an evaluation of fitness for anaesthesia, assuming the anaesthesia is the most life-threatening process to the patient. On the contrary, when performed carefully with appropriate monitoring and timely interventions, the period of anaesthesia represents a period of relative stability for the patient in the vast majority of time. Rather, preoperative risk assessment evaluates the capacity of the patient to withstand the acute physiological perturbations resulting from the entire operative period that extends well into the recovery phase. The critical element is to estimate whether the patient can meet the increased oxygen demand due to the acute stress response to surgery. Therefore, the assessment tends to focus upon the cardiac and respiratory system as these are critical determinants of oxygen supply to tissues. Another point of focus of the examination is conditions affecting the level of consciousness, whether it involves the central nervous system or secondary to metabolic disturbances. Acute delirium is associated with high perioperative morbidity and mortality. Delayed emergence from anaesthesia may occur in patients suffering from preoperative delirium. Alternatively, the effects of general anaesthesia may further contribute to the delirious state, complicating the clinical picture.

\section{Pulmonary risk stratification}

Risk factors for developing postoperative pulmonary complications

In a systematic review of more than 100 studies, the authors identified patient, procedure and laboratory related risk factors for the development of postoperative pulmonary complications in non-cardiothoracic surgery that were supported by good evidence. Those of interest to the fracture hip population include advanced age, American Society of Anesthesiologists class 2 or higher, functional dependence, chronic obstructive pulmonary disease and congestive heart failure, emergency surgery, general anaesthesia, prolonged surgery and serum albumin level less than $30 \mathrm{~g} / \mathrm{L}$. Interestingly, for the study population there was insufficient evidence to support preoperative spirometry as a tool to stratify risk [4]. Similar risk factors have also been incorporated into a respiratory failure risk index [5].The presence of any of these conditions should alert the primary treating doctors to request for an early anaesthetic consultation. 
Postoperative pulmonary complications: why does it occur?

Severe factors can individually or in combination precipitate respiratory failure should the patient fail to increase and sustain the necessary minute ventilation. The basal requirement for oxygen consumption is elevated postoperatively from an average of $110 \mathrm{ml} / \mathrm{min} / \mathrm{m}^{2}$ at rest to up to an average of $170 \mathrm{ml} / \mathrm{min} / \mathrm{m}^{2}$ from systemic inflammatory response to major surgery $[6,7]$. Also, other intervening factors such as fever or sepsis can further increase oxygen demand and carbon dioxide production. Atelectasis is common after general anaesthesia [8] and even after spinal anaesthesia [9] and will contribute to ventilation perfusion mismatch and resultant hypoxemia. Sedative effects from subanaesthetic doses of inhalational agents or opioid analgesia can depress respiration and the ability of the body to oxygenate the blood and eliminate carbon dioxide. The urge to cough can be depressed by opioid analgesics, together with the impaired mucociliary clearance mechanism of the respiratory epithelium from general anaesthesia [10] can predispose the patient to develop pneumonia. Therefore, the anaesthesiologist has to evaluate the likelihood the patient can adequately compensate for these adverse factors by increasing their respiratory effort without developing exhaustion.

Preoperative pulmonary assessment: what do we look for?

In the preoperative evaluation of pulmonary risk, the anaesthesiologist is required to determine the likelihood in the postoperative period that the patient can adequately oxygenate the blood, eliminate carbon dioxide, cough adequately to expel lung secretions and to meet the increased oxygen demand. Clinical assessment is of paramount importance although not always possible from the uncooperative patient; however, much information can still be gleaned from the patient's general appearance. Those who appear frail, pale, cyanotic and tachypneic are less likely to sustain a prolonged increase respiratory effort. Certain physiological parameters may give an indication of the likelihood of developing postoperative pulmonary complications. Room-air saturation of below $90 \%$ represents an important finding as from this point a small decrement of partial pressure will lead to a large decrease in saturation. Those with low haemoglobin will have a reduced oxygen carrying capacity. Some objective parameters may be associated with the possibility of $\mathrm{CO}_{2}$ retention. These include a reduced $\mathrm{FEV}_{1}$ of between $27 \%$ and $47 \%$ of predicted [11, 12], forced vital capacity of less than $1.7 \mathrm{~L}$ [13]. A patient with a peak expiratory flow rate of less than $82 \mathrm{~L} / \mathrm{min}$ would probably have difficulty generating an effective cough to clear sputum [14]. An estimation of the patient's maximal breathing capacity (MBC) in comparison to the patient's baseline minute volume may provide an insight into their respiratory reserve. The MBC may be approximated by multiplying their $\mathrm{FEV}_{1}$ by 35 , with healthy people being able to sustain a minute volume of $50 \%$ to $60 \%$ of their MBC $[15,16]$.

Acute chest infection or exacerbation of chronic lung condition presents a dilemma as the condition may or may not be improved with ongoing immobility. A decision would have to be made on an individual basis depending on the assessment of their pulmonary reserve. Thankfully, the operative site of a fractured hip is well away from respiratory muscles and by itself is unlikely to interfere with breathing in the postoperative period unlike thoracic or abdominal surgery. Patients with marginal pulmonary reserves may still proceed to surgery provided there is adequate availability of postoperative monitoring, pulmonary rehabilitation and ventilator support if required.

\section{Preoperative cardiac risk stratification}

The use of consensus guidelines

Excellent guidelines are available to assist with preoperative cardiac risk evaluation and decision making [17, 18]; however, it is recognized that there may be times when difficulties may arise in following these guidelines. There may be differences in availability of expertise or resources in different institutions. There may also be patient-related limitations such as difficulty in obtaining an accurate functional status from elderly patients with limited mobility. They may not be stressed to the point of cardiac ischemia in their daily life and is therefore "asymptomatic". Nevertheless, the spirit of the guidelines should apply and is summed up in this statement: "The overriding theme of this document is that intervention is rarely necessary to simply lower the risk of surgery unless such intervention is indicated irrespective of the preoperative context. The purpose of preoperative evaluation is not to give medical clearance but rather to perform an evaluation of the patient's current medical status; make recommendations concerning the evaluation, management, and risk of cardiac problems over the entire perioperative period; and provide a clinical risk profile that the patient, primary physician and non-physician caregivers, anaesthesiologist, and surgeon can use in making treatment decisions that may influence short- and long-term cardiac outcomes. No test should be performed unless it is likely to influence patient treatment. The goal of the consultation is the optimal care of the patient."[18] 
Important cardiac conditions requiring evaluation

Accordingly, those with unstable coronary syndromes, such as unstable or severe angina or a recent myocardial infarction (7 days to 1 month), decompensated heart failure, significant arrhythmias (including supraventricular arrhythmias with ventricular rate above 100 , high-grade atrioventricular heart blocks) and severe valvular disease should undergo cardiac evaluation. Evaluation should also be performed where uncertainty exists over the diagnosis (e.g. dyspnoea of unknown origin) and for those with pacemakers (to review its indication, evaluate the battery life and resetting the mode if indicated). The purpose of these consultations is to confirm diagnosis, delineate the severity of the disease and whether there is any room for improvement with medical treatment in light of the clinical findings and not to obtain a medical clearance for anaesthesia from our physician colleagues. Where timely preoperative intervention may not be available, the information gathered may influence the postoperative management of the patient such as the patient disposition post surgery or postoperative coronary interventions. Current evidence would indicate there is no benefit in delaying surgery for patients with mild to moderate hypertension (systolic less than $180 \mathrm{mmHg}$ and diastolic less than $110 \mathrm{mmHg}$ ) [19, 20]. The evidence for severe hypertension is less clear, but the decision should be based on the duration of the hypertension and whether end organ damage is present. Conditions that are casually linked to hypertension such as heart failure, coronary artery disease, renal impairment and cerebrovascular accident constitute important components of the revised cardiac risk index and their presence would independently elevate cardiac risk [21].

Patients receiving anti-platelet therapy

Management of the patient on anti-platelet agents such as clopidogrel for drug-eluting coronary stents is difficult and controversial. The withdrawal of these agents represents a major risk factor for thrombosis for all types of stents, particularly for late stent thrombosis in drug-eluting stents $[22,23]$. There are patients who are at particular risk, including those with a history of stent thrombosis, patients with multiple stent, long stents or stents placed at a bifurcation, incomplete revascularization, diabetic patients or patients with a low ejection fraction [23]. In general, neuroaxial anaesthesia is contraindicated in patients taking these medications (except those taking aspirin alone) but not for general anaesthesia. Some patients may be less tolerant of increased blood loss associated with these agents and special arrangements may have to be made for these situations that mandate a multidisciplinary approach, with considerations of implementing bridging anticoagulation therapy if warranted [24].

\section{Central nervous system evaluation}

Delirium is common in hospitalised patients [25] and is common in those with pre-existing cognitive impairment [26]. It may develop in up to half of patients postoperatively, especially after hip and vascular surgery [27, 28]. Postoperative delirium is associated with increased morbidity and mortality and increases length of stay $[29,30]$. Therefore, the presence of delirium preoperatively warrants prompt investigations, but this condition is often unrecognized [31]. Of importance is to rule out major life-threatening conditions such as hypoxia, hypoglycaemia, major fluid and electrolyte imbalances, sepsis and major organ impairment. If suggested by corroborative history and/or physical signs, neuroimaging of the head may be needed to rule out a cerebrovascular accident. A recent stroke is accompanied by impaired autoregulation of cerebral vessels and haemodynamic stress associated with surgery and general anaesthesia can potentially make the penumbra area susceptible to haemorrhagic transformation and worsening ischemia extend the infarct area. There is no consensus as to the period of vulnerability, but it may be in the order of 2 weeks [32].

\section{When to proceed and when to defer?}

A good rule of thumb when considering whether to proceed with operative treatment is to determine whether there are conditions present that may be detrimental or even lifethreatening that require medical treatment in its own right in the absence of surgery. Such conditions may include dehydration with acute renal impairment, severe electrolyte abnormalities (a sodium or potassium level outside the range of 120 to $150 \mathrm{mmol} / \mathrm{L}$ and 2.8 to $6.0 \mathrm{mmol} / \mathrm{L}$, respectively), symptomatic anaemia and uncontrolled diabetes with risk of developing dehydration from polyuria or hyperosmolar coma. In addition, one would consider delaying surgery for unfasted patients and to correct any correctable coagulopathy and anaemia. The level at which this occurs should ideally be individualised, but transfusion should be considered when preoperative haemoglobin level is between 7 and $10 \mathrm{~g} / \mathrm{dL}$. Operation should only be deferred if there is a reasonable likelihood of improving the conditions that are precluding surgery. To optimize, as defined by the Oxford Dictionary, is to "make the best or most effective use of a situation or resource". Optimization is what we hope to achieve for every preoperative patient; however, there are times when the best a patient can achieve still places him or her in a high-risk category, 
despite having achieved certain objective criteria. If there are no further improvements possible without subjecting the patient to other stressful procedures, a decision has to be made to either proceed with operative or conservative treatment. Prolonged or repeated fasting orders during periods of decisional uncertainty can only cause further harm to patients. Many intervening factors, medical or nonmedical, may wade into the decision to operate or not. Ultimately, each case have to be considered on its own merit and communication between surgeons, anaesthesiologists, physicians, intensive care physicians and the patient is paramount in decision making.

\section{Why then does last minute cancellation occur?}

Last minute cancellation or undue delay of an operation due to medical reasons is frustrating to all concerned as it is mostly avoidable and is costly to both the patient and the health care system. It frequently occurs consequent to expectation differences and breakdown in communication between the physicians from different disciplines involved. The development of institutional guidelines on the management of fractured hip patients (see Fig. 1) that is followed from the time the diagnosis is first suspected would bypass much of the uncertainty regarding expectations of what need
Fig. 1 An example of an institutional guideline on the management of hip fracture patients. Ix = Investigations; $\mathrm{CBC}=$ Complete Blood Count; $\mathrm{Na}=$ Sodium; $\mathrm{K}=$ Potassium; $\mathrm{Ur}=\mathrm{Urea} ; \mathrm{Cr}=$ Creatinine; Glu = Glucose; LFT = Liver Function Tests; PT = Prothrombin Time; APTT $=$ Activated Partial Thromoplastin Time; $\mathrm{CK}=$ Creatine Kinase; TFT $=$ Thyroid Function Test; IV = Intravenous; $\mathrm{CXR}=$ Chest $\mathrm{X}$ ray; $\mathrm{CT}=$ Computerised Tomography; $\mathrm{CVA}=$ Cerebrovascular Accident; $\mathrm{OT}=$ Operating Theatre; $\mathrm{COPD}=$ Chronic Obstructive Pulmonary Disease; IHD = Ischaemic Heart Disease $; \mathrm{AMI}=$ Acute Myocardial Infarction

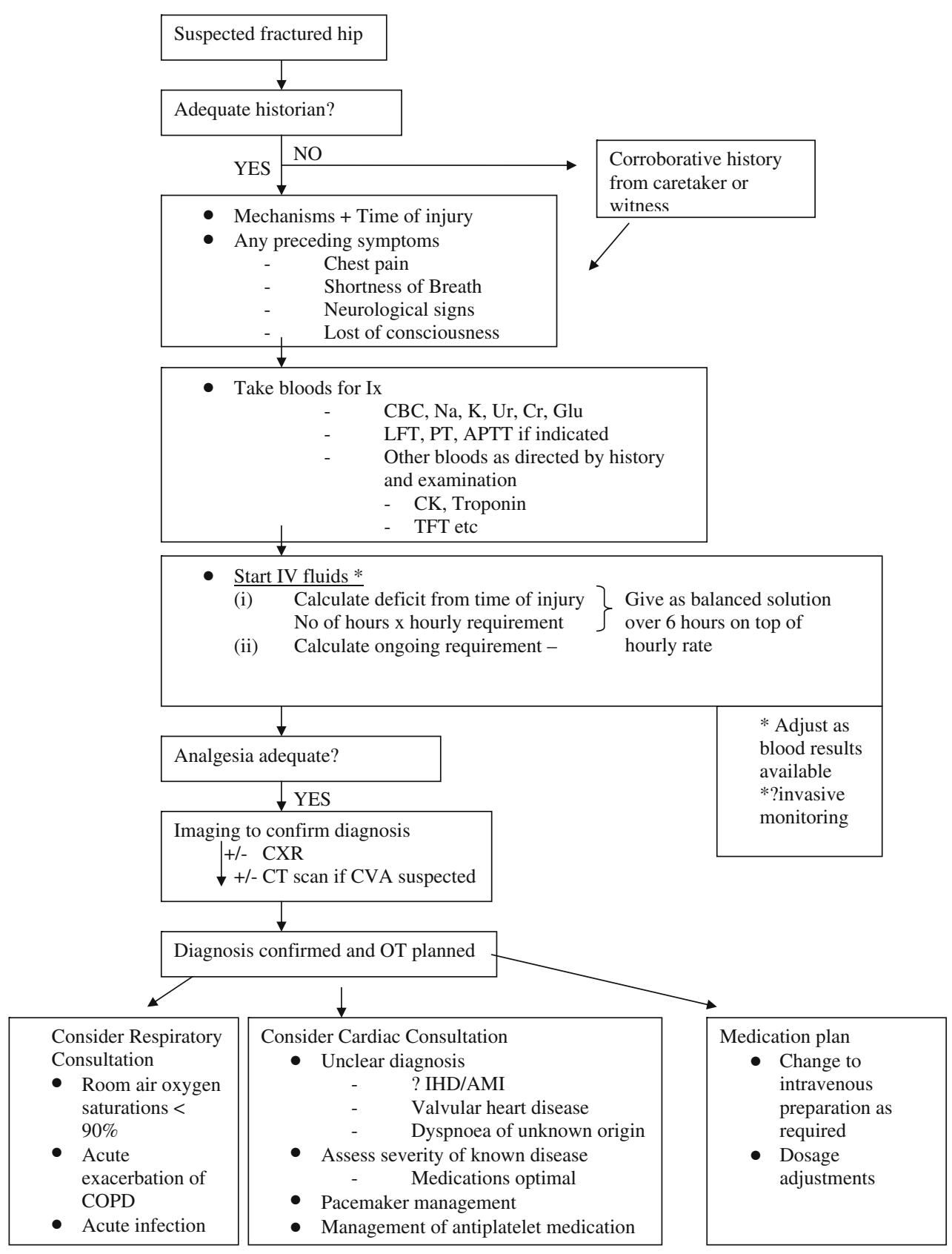


to be achieved for the patient before surgery is considered. Invariably "one size does not fit all", and individual circumstances may require deviations from set guidelines; however, it would substantially reduce delay as the indicated investigations and referrals would immediately be made. Lastly, such guidelines must be individualised to specific institutions or area health and require the input of all specialities involved and be reviewed and audited on regular intervals to ensure it is effective in achieving its aims.

Conflicts of interest The authors declare that there are no conflicts of interest.

Open Access This article is distributed under the terms of the Creative Commons Attribution Noncommercial License which permits any noncommercial use, distribution, and reproduction in any medium, provided the original author(s) and source are credited.

\section{References}

1. Price JD, Sear JW, Venn RM (2004) Perioperative fluid volume optimization following proximal femoral fracture. Cochrane Database Syst Rev 1:CD003004

2. Devereaux PJ, Goldman L, Cook DJ, Gilbert K, Leslie K, Guyatt GH (2005) Perioperative cardiac events in patients undergoing noncardiac surgery: a review of the magnitude of the problem, the pathophysiology of the events and methods to estimate and communicate risk. CMAJ 173:627-634

3. Sorensen JV, Rahr HB, Jensen HP, Borris LC, Lassen MR, Ejstrud P (1992) Markers of coagulation and fibrinolysis after fractures of the lower extremities. Thromb Res 65:479-486

4. Smetana GW, Lawrence VA, Cornell JE, American college of Physicians (2006) Preoperative pulmonary risk stratification for noncardiothoracic surgery: systematic review for the American college of physicians. Ann Intern Med 144:581-595

5. Arozullah AM, Daley J, Henderson WG, Khuri SF (2000) Multifactorial risk index for predicting postoperative respiratory failure in men after major noncardiac surgery. The national veterans administration surgical quality improvement program. Ann Surg 232:242-253

6. Older P, Smith R (1988) Experience with the preoperative invasive measurement of haemodynamic, respiratory and renal function in 100 elderly patients scheduled for major abdominal surgery. Anaesth Intensive Care 16:389-395

7. Shoemaker WC, Appel PL, Kram HB, Waxman K, Lee TS (1988) Prospective trial of supranormal values of survivors as therapeutic goals in high-risk surgical patients. Chest 94:1176-1186

8. Magnusson L, Spahn DR (2003) New concepts of atelectasis during general anaesthesia. Br J Anaesth 91:61-72

9. Egbert LD, Tamersoy K, Navy T, Deas TC (1961) Pulmonary function during spinal anesthesia: the mechanism of cough depression. Anesthesiology 22:882-885

10. Gamsu G, Singer MM, Vincent HH, Berry S, Nadel JA (1976) Postoperative impairment of mucous transport in the lung. Am Rev Respir Dis 114:673-679

11. Scano G, Spinelli A, Duranti R, Gorini M, Gigliotti F, Goti P, MilicEmili J (1995) Carbon dioxide responsiveness in COPD patients with and without chronic hypercapnia. Eur Respir J 8:78-85
12. Cloosterman SG, Hofland ID, van Schayck CP, Folgering HT (1998) Exertional dyspnoea in patients with airway obstruction, with and without $\mathrm{CO} 2$ retention. Thorax 53:768-774

13. Montes de Oca M, Celli BR (1998) Mouth occlusion pressure, $\mathrm{CO}_{2}$ response and hypercapnia in severe chronic obstructive pulmonary disease. Eur Respir J 12:666-671

14. Smina M, Salam A, Khamiees M, Gada P, Amoateng-Adjepong Y, Manthous CA (2003) Cough peak flows and extubation outcomes. Chest 124:262-268

15. Zocche GP, Fritts HW Jr, Cournand A (1960) Fraction of maximum breathing capacity available for prolonged hyperventilation. J Appl Physiol 15:1073-1074

16. Melissant CF, Lammers JW, Demedts M (1998) Relationship between external resistances, lung function changes and maximal exercise capacity. Eur Respir J 11:1369-1375

17. Poldermans D, Bax JJ, Boersma E, De Hert S, Eeckhout E, Fowkes G, Gorenek B, Hennerici MG, Iung B, Kelm M, Kjeldsen KP, Kristensen SD, Lopez-Sendon J, Pelosi P, Philippe F, Pierard L, Ponikowski P, Schmid JP, Sellevold OF, Sicari R, Van den Berghe G, Vermassen F, Hoeks SE, Vanhorebeek I (2009) Task force for preoperative cardiac risk A, perioperative cardiac management in non-cardiac surgery $\mathrm{ESoC}$, European society of A. Guidelines for pre-operative cardiac risk assessment and perioperative cardiac management in non-cardiac surgery: the task force for preoperative cardiac risk assessment and perioperative cardiac management in non-cardiac surgery of the European society of cardiology (ESC) and endorsed by the European society of anaesthesiology (ESA). Eur Heart J 30:2769-2812

18. Fleisher LA, Beckman JA, Brown KA, Calkins H, Chaikof EL, Fleischmann KE, Freeman WK, Froehlich JB, Kasper EK, Kersten JR, Riegel B, Robb JF (2009) 2009 ACCF/AHA focused update on perioperative beta blockade incorporated into the ACC/ AHA 2007 guidelines on perioperative cardiovascular evaluation and care for noncardiac surgery: a report of the American College of Cardiology Foundation/American Heart Association task force on practice guidelines. Circulation 120:e169-e276

19. Chobanian AV, Bakris GL, Black HR, Cushman WC, Green LA, Izzo JL Jr, Jones DW, Materson BJ, Oparil S, Wright JT Jr, Roccella EJ (2003) National heart 1, blood institute joint national committee on prevention $\mathrm{DE}$, treatment of high blood pressure, national high blood pressure education program coordinating committee. The seventh report of the joint national committee on prevention, detection, evaluation, and treatment of high blood pressure: the JNC 7 report. JAMA 289:2560-2572

20. Fleisher LA, Beckman JA, Brown KA, Calkins H, Chaikof E, Fleischmann KE, Freeman WK, Froehlich JB, Kasper EK, Kersten JR, Riegel B, Robb JF, Acc/Aha Task Force M, Smith SC Jr, Jacobs AK, Adams CD, Anderson JL, Antman EM, Buller CE, Creager MA, Ettinger SM, Faxon DP, Fuster V, Halperin JL, Hiratzka LF, Hunt SA, Lytle BW, Md RN, Ornato JP, Page RL, Tarkington LG, Yancy CW (2007) ACC/AHA 2007 guidelines on perioperative cardiovascular evaluation and care for noncardiac surgery: executive summary: a report of the American College of Cardiology/American Heart Association task force on practice guidelines (writing committee to revise the 2002 guidelines on perioperative cardiovascular evaluation for noncardiac surgery): developed in collaboration with the American Society of Echocardiography, American Society of Nuclear Cardiology, Heart Rhythm Society, Society of Cardiovascular Anesthesiologists, Society for Cardiovascular Angiography and Interventions, Society for Vascular Medicine and Biology, and Society for Vascular Surgery. Circulation 116:1971-1996

21. Lee TH, Marcantonio ER, Mangione CM, Thomas EJ, Polanczyk CA, Cook EF, Sugarbaker DJ, Donaldson MC, Poss R, Ho KKL, Ludwig LE, Pedan A, Goldman L (1999) Derivation and 
prospective validation of a simple index for prediction of cardiac risk of major noncardiac surgery. Circulation 100:1043-1049

22. Rodriguez AE, Mieres J, Fernandez-Pereira C, Vigo CF, RodriguezAlemparte M, Berrocal D, Grinfeld L, Palacios I (2006) Coronary stent thrombosis in the current drug-eluting stent era: insights from the ERACI III trial. J Am Coll Cardiol 47:205-207

23. Iakovou I, Schmidt T, Bonizzoni E, Ge L, Sangiorgi GM, Stankovic G, Airoldi F, Chieffo A, Montorfano M, Carlino M, Michev I, Corvaja N, Briguori C, Gerckens U, Grube E, Colombo A (2005) Incidence, predictors, and outcome of thrombosis after successful implantation of drug-eluting stents. JAMA 293:2126-2130

24. Albaladejo P, Marret E, Piriou V, Samama C-M (2006) Perioperative management of antiplatelet agents in patients with coronary stents: recommendations of a French task force. Br J Anaesth 97:580-582

25. Trzepacz PT (1996) Delirium. Advances in diagnosis, pathophysiology, and treatment. Psychiatr Clin North Am 19:429-448

26. Practice guideline for the treatment of patients with delirium (1999) American Psychiatric Association. Am J Psychiatry 156:1-20
27. Marcantonio ER, Flacker JM, Michaels M, Resnick NM (2000) Delirium is independently associated with poor functional recovery after hip fracture. J Am Geriatr Soc 48:618-624

28. Sasajima Y, Sasajima T, Uchida H, Kawai S, Haga M, Akasaka N, Kusakabe M, Inaba M, Goh K, Yamamoto H (2000) Postoperative delirium in patients with chronic lower limb ischaemia: what are the specific markers? Eur J Vasc Endovasc Surg 20:132-137

29. Mullen JO, Mullen NL (1992) Hip fracture mortality. A prospective, multifactorial study to predict and minimize death risk. Clin Orthop Relat Res 280:214-22

30. Nightingale S, Holmes J, Mason J, House A (2001) Psychiatric illness and mortality after hip fracture. Lancet 357:1264-1265

31. Inouye SK (1994) The dilemma of delirium: clinical and research controversies regarding diagnosis and evaluation of delirium in hospitalized elderly medical patients. Am J Med 97:278-288

32. Blacker DJ, Flemming KD, Link MJ, Brown RD Jr (2004) The preoperative cerebrovascular consultation: common cerebrovascular questions before general or cardiac surgery. Mayo Clin Proc $79: 223-229$ 